\title{
A Hands-Free Ride
}

\section{I recently had the opportunity to take a ride in a Waymo self-driving car in Chandler, AZ. I had been looking forward to this experience, not only to see how well the technologyworked}

but also what the experience might be like as a passenger. Upon my arrival at the Waymo facility, I had apparently approached the side of the building where the Waymo cars go at the end of their duty cycles to be refueled and inspected. As I drove in, I was more or less surrounded by incoming Waymo vehicles. I relaxed as they navigated their way around me.

I was not unaccompanied on this test drive. There was an emergency backup driver at the wheel who did not need to take any actions during the 15-minute drive. In addition, I had an engineer with me who was able to show what the Waymo car's "brain" (my term) was seeing on the road. I also had a business operations escort who was able to answer questions about how this self-driving ride service was being rolled out. The rider in the back seat has a display showing the traffic nearby and providing information, alerts, opportunity to speak with a customer service person, and so on. Knowing what the car "sees" is reassuring.

Our merry band of four took off for a residential neighborhood. There were a number of challenging situations to deal with. One that impressed me mightily was a left turn at a twoway intersection with no traffic signal. It was a fairly busy road and I would have found it challenging to estimate when it was safe to turn had I been at the wheel. A second interesting maneuver involved a left turn at a traffic signal that included both a red light (to prevent traffic from advancing into the intersection to cross the street) and a flashing yellow arrow that meant "turn left when it is safe to do so, proceed with caution." Both of these turns were made successfully.

In earlier experiences, I felt the car tended to ride somewhat unevenly as if the "driver" were prone to lightly tapping the brake. This ride seemed much smoother and more natural. We cruised around the residential neighborhood, negotiating a traffic circle, four-way intersections, cross traffic, pedestrians, a school area, and speed bumps. Our Waymo car negotiated all of these situations without apparent difficulty. I learned the cars have access to an extremely detailed amount of information about

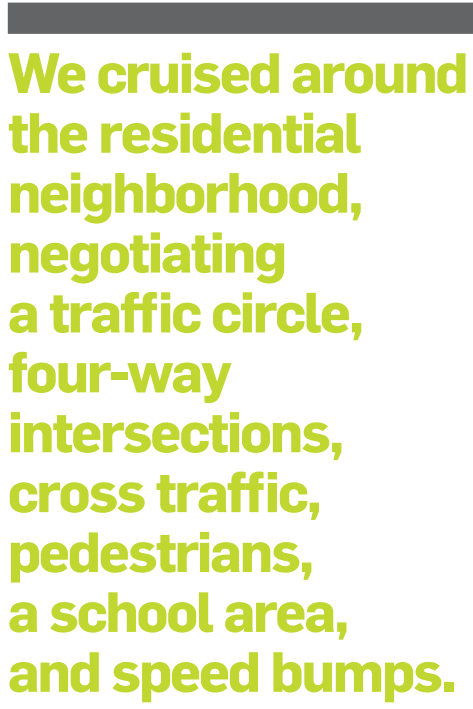

the area in which they are operating so the sensor data obtained in real time is combined with known details of local conditions (such as speed bumps) to aid in decision making.

The car I was in displayed speed in $\mathrm{km} /$ hour but I didn't know that and momentarily panicked when I thought we were exceeding the posted mph speed limit! As my vehicle continued on its way, negotiating street crossings and turns, I marveled at the natural way it navigated in normal city traffic. The science of autonomous driving has come a very long way from the early grand challenge posed 15 years ago by the Defense Advanced Research Projects Agency. From those early beginnings, Waymo cars have driven millions of miles in city traffic and billions of miles in simulation.

Looking back on this experience, my sense of assurance that these cars really are equipped to handle unusual or at least complex traffic situations rose significantly. The level of care for safety that Waymo has taken has been documented in its reports to the U.S. Department of Transportation and the National Transportation Safety Board. This personal experience on the ground (err, road) reinforced my belief that self-driving car service is demonstrably feasible, especially in areas where weather conditions are favorable to its operation.

Vinton G. Cerf is vice president and Chief Internet Evangelist at Google. He served as ACM president from 2012-2014. 